\title{
Hyperthyroidism and psychiatric morbidity: evidence from a Danish nationwide register study
}

\author{
Frans Brandt, Marianne Thvilum, Dorthe Almind', Kaare Christensen ${ }^{1,2,3}$, \\ Anders Green ${ }^{4}$, Laszlo Hegedüs and Thomas Heiberg Brix
}

Department of Endocrinology and Metabolism, Odense University Hospital, Sdr. Boulevard 29, 5000 Odense C, Denmark, ${ }^{1}$ The Danish Aging Research Center and The Danish Twin Registry, University of Southern Denmark, J.B. Winsløwsvej 9b, 5000 Odense C, Denmark, ${ }^{2}$ Department of Clinical Genetics, Odense University Hospital, Sdr. Boulevard 29, 5000 Odense C, Denmark, ${ }^{3}$ Department of Clinical Biochemistry and Pharmacology, Odense University Hospital, Sdr. Boulevard 29, 5000 Odense C, Denmark and ${ }^{4}$ Odense Patient Data Exploratory Network, Institute of Clinical Research, University of Southern Denmark, J. B. Winsløwsvej 9A, 5000 Odense C, Denmark
Correspondence should be addressed to F Brandt Email

frans.hjelm.brandt. kristensen@rsyd.dk

\section{Abstract}

Objective: Thyroid hormones are essential for the normal development of the fetal brain, while hyperthyroidism in adults is associated with mood symptoms and reduced quality of life. In this study, we aimed to investigate the association and temporal relationship between hyperthyroidism and psychiatric morbidity.

Design: Register-based nationwide cohort study.

Method: Data on hyperthyroidism and psychiatric morbidity were obtained by record linkage of the Danish National Patient Registry and the Danish National Prescription Registry. A total of 2631 hyperthyroid individuals were identified and matched 1:4 with non-hyperthyroid controls and followed for a mean duration of 6 years (range 0-13). Logistic and Cox regression models were used to assess the risk of psychiatric morbidity before and after the diagnosis of hyperthyroidism respectively. Results: Before the diagnosis of hyperthyroidism, such individuals had an increased risk of being hospitalized with psychiatric diagnoses (odds ratio (OR): 1.33; 95\% Cl: 0.98-1.80) and an increased risk of being treated with antipsychotics (OR: 1.17; 95\% Cl: 1.00-1.38), antidepressants (OR: 1.13; 95\% Cl: 1.01-1.27), or anxiolytics (OR: 1.28; 95\% Cl: 1.16-1.42). After the diagnosis of hyperthyroidism, there was a higher risk of being hospitalized with psychiatric diagnoses (hazard ratio (HR): 1.51; 95\% Cl: 1.11-2.05) and an increased risk of being treated with antipsychotics (HR: 1.46; 95\% Cl: 1.20-1.79), antidepressants (HR: 1.54; 95\% Cl: 1.36-1.74), or anxiolytics (HR: 1.47; 95\% Cl: 1.27-1.69).

Conclusions: Hyperthyroid individuals have an increased risk of being hospitalized with psychiatric diagnoses and being treated with antipsychotics, antidepressants, and anxiolytics, both before and after the diagnosis of hyperthyroidism.

\section{Introduction}

Thyroid hormones have major effects on developmental and physiological processes and the CNS is one of the most important targets (1). In the brain, thyroxine is converted to the more metabolically active triiodothyronine $\left(T_{3}\right)$ by the type II deiodinases (D2) and to the inactive reverse $\mathrm{T}_{3}\left(\mathrm{rT}_{3}\right)$ by the type III deiodinases $(2,3)$. A reduced supply of thyroid hormones in developing humans can cause irreversible cognitive impairment and neurological
(C) 2014 European Society of Endocrinology Printed in Great Britain deficits (4). In adults, the effect of thyroid dysfunction on mental and brain functions is less clear-cut. However, mood disorders and decreased quality of life are often prominent in hyperthyroid patients, also after restoration of euthyroidism (5). Moreover, thyroid hormones interact with neurotransmitters such as serotonin and norepinephrine (6), both of which are involved in the pathway of developing psychiatric diseases $(7,8)$, making 
the link between hyperthyroidism and psychiatric morbidity plausible.

Based on nationwide data with long-term follow-up and adjustment for pre-existing co-morbidity, we have demonstrated an increased burden of somatic morbidity, both before and after the diagnosis of thyroid dysfunction $(9,10)$. Not surprisingly, this is followed by excess mortality in patients with hypothyroidism $(11,12)$ as well as hyperthyroidism $(13,14,15)$. Psychiatric morbidity, suggested to be associated with thyroid dysfunction $(16,17,18,19,20)$, most probably also contributes to this increase in mortality. Although an association between thyroid dysfunction and psychiatric conditions has been suggested since the earliest descriptions of hypothyroidism as well as hyperthyroidism (21), our current knowledge is limited. In essence, the literature regarding the relationship between thyroid dysfunction and psychiatric disorders is ambiguous and almost entirely based on underpowered studies with heterogenic definitions of both psychiatric and thyroid diseases $(16,17,18,19,20$, $22,23,24,25)$. Also, when based on an adequate sample size, the studies report divergent findings regarding the association between hyperthyroidism and psychiatric morbidity $(20,24)$. While Thomsen et al. (20) reported a 3.6 times increased risk of hospitalization with affective disorders in patients with hyperthyroidism, Engum et al. (24) found no association between biochemical hyperthyroidism and symptoms of depression and anxiety. In addition, most of the existing studies are cross-sectional and were not designed to examine the temporal relationship between hyperthyroidism and psychiatric morbidity $(17,18,19,20,25)$. Not to be neglected, drugs used in the treatment of some psychiatric diseases could also influence the development of hyperthyroidism; for example, tricyclic antidepressants (TCAs) have been suggested to complex with iodine, inhibit D2, and trigger thyroid autoimmunity (26). In line with this, psychiatric diseases have been reported to be associated with an increased risk of subsequent hospitalization with hyperthyroidism (27). Another shortcoming is the ignoring of possible genetic confounding. Employing twin analyses, we have previously shown that the association between hyperthyroidism and excess mortality could be influenced by genetic confounding (14). As hyperthyroidism $(28,29)$ and various psychiatric disorders $(30,31,32)$ demonstrate familial aggregation, they could be co-inherited.

To overcome some of the shortcomings of previous studies, in this study, we used data from Danish nationwide health registers on hospitalization and pharmacological treatment to evaluate the temporal relationship between hyperthyroidism and psychiatric morbidity. Using population-based twin data, we further examined whether this association was influenced by genetic confounding.

\section{Subjects and methods}

\section{Subjects}

Subjects included in this study were identified by record linkage of Danish health registers. A detailed description of the ascertainment procedure and statistical methods as well as all the registers utilized has been given elsewhere $(14,15)$.

In brief, from a random 5\% sample of the Danish background population, 2631 singleton individuals with incident hyperthyroidism, recorded between 1996 and 2008, were identified and matched 1:4 with control subjects according to age and sex, following the principles of density sampling $(14,15)$. From the Danish Twin Registry, we identified 375 same-sex twin pairs discordant for hyperthyroidism. All the subjects were followed until migration, death, or December 31, 2008.

\section{Diagnosis of hyperthyroidism}

Information on thyroid status was drawn from the Danish National Patient Registry (DNPR) or the Danish National Prescription Registry (DNPrR). To be classified as having hyperthyroidism, subjects should be registered in either the DNPR or the DNPrR. In the DNPR, hyperthyroidism was defined by the International Classification of Diseases (ICD) ten codes E05-E05.9. In the DNPrR, hyperthyroidism was defined by at least two dispensed prescriptions of anti-thyroid medications $(\mathrm{ATC}=\mathrm{H} 03 \mathrm{~B})$. Date of the onset of hyperthyroidism (index date) was chosen as the first date of registration in either the DNPR or the DNPrR.

\section{Psychiatric morbidity}

The overall outcomes were psychiatric morbidity as identified from the DNPR and treatment with psychiatric drugs as identified from the DNPrR between 1995 and 2008. In the DNPR, diagnostic codes include a principal diagnosis reflecting the primary cause of admission and up to 19 secondary discharge diagnoses. As an example, a patient can be hospitalized primarily due to pneumonia, but may also suffer from psychiatric disease. In this case, 
psychiatric disease is a secondary diagnosis. On the other hand, an individual could be hospitalized due to the onset or worsening of psychiatric disease, making it the primary cause of hospitalization. In the DNPrR, coding for medical products sold with a prescription is according to the Anatomical Therapeutic Chemical (ATC) classification system. Thus, psychiatric morbidity was identified either based on treatment in a hospital setting, including hospital outpatient clinics, or by the use of psychiatric medication (antipsychotics, antidepressants, or anxiolytics), with a minimum of one prescription. All relevant ICD codes as well as ATC codes are given in Table 1. Both the DNPR and DNPrR have been used in other Danish register-based surveys studying psychiatric morbidity, e.g. based on the use of benzodiazepines, antipsychotics, and antidepressants $(20,27,33,34,35)$. For each individual, the first date of possible registration in each of these outcome groups was identified. Accordingly, we stratified for the first registration in each group before and after the date of the diagnosis of hyperthyroidism.

\section{Statistical analyses}

Group frequencies were compared with using Pearson's $\chi^{2}$ test, whereas group means were compared using a $t$-test.

The odds ratio (OR) for the prevalence of psychiatric morbidity, before the diagnosis of hyperthyroidism, was evaluated in a logistic regression analysis adjusted for age and sex. The relationship between the first registration of hyperthyroidism and the subsequent first registration of psychiatric morbidity was evaluated using a Cox regression analysis. Age was chosen as the underlying time variable. In both singleton cases and controls, person-years of follow-up were accumulated from the index date of the case and were terminated at the date of the diagnosis of morbidity in the relevant disease category, migration, death, or end of follow-up (December 31, 2008), whichever came first. In all the Cox analyses, the variable 'pair' was used as a stratum variable, fixing the baseline hazard within a matched pair, while at the same time allowing this baseline hazard to vary freely between pairs. Afterwards, all the Cox regression analyses were adjusted for the degree of somatic co-morbidity preceding the diagnosis of hyperthyroidism, using the Charlson score (CS). Moreover, the educational level was taken into consideration, categorized into primary, secondary, or tertiary, as described previously (36). The CS accounts for 19 various disease categories (myocardial infarction, heart failure, vascular disease, cerebrovascular disease, dementia, chronic lung disease, rheumatic disease, gastric ulcer, liver disease, diabetes mellitus without complications, diabetes mellitus with complications, hemiplegia, kidney disease, cancer, cancer with metastases, lymphoma, leukemia, liver failure, and AIDS). It allows for the calculation of an individual 1-year mortality risk depending on the degree and type of co-morbidity $(14,15)$.

Due to the register-based design, we cannot exclude that the registration of one disease might lead to a higher risk of registration with other diseases due to more intense medical evaluation. To quantify this possible bias, we repeated all the analyses but censored diagnoses made 365 days before and after the diagnosis of hyperthyroidism. In addition, since hyperthyroidism is associated with increased mortality $(13,14,15)$, cases, at least theoretically, have a shorter observation period than the controls and thus a lower risk of being diagnosed with psychiatric morbidity. To account for this influence of competing risk, the risk of psychiatric morbidity was also evaluated by the method of Fine and Gray (15).

In the twin population, we carried out intra-pair analyses, in which the hyperthyroid twin was matched with the corresponding euthyroid co-twin. In these analyses, all the psychiatric disease groups, hospital treatment, and medications were handled as one group, and disease frequency was evaluated by conditional logistic regression analyses. All the twin analyses were stratified for zygosity.

Significant differences were defined as a $P$ value below 0.05, using two-tailed tests. All the analyses were conducted using STATA version 11.0 (2009; Stata Corporation, College Station, TX, USA).

Table 1 Definition of psychiatric morbidity from data in the Danish National Patient Registry and the Danish National Prescription Registry.

\begin{tabular}{|c|c|c|}
\hline Outcome & DNPR $^{a}$ & DNPrR $^{\mathrm{b}}$ \\
\hline $\begin{array}{l}\text { Hospitalization with } \\
\text { psychiatric diagnoses }\end{array}$ & $\begin{array}{l}\text { DF20-25, } \\
\text { DF28-34, } \\
\text { DF38-39, } \\
\text { DF41-41.9 }\end{array}$ & \\
\hline Treatment with antipsychotics & - & N05A \\
\hline $\begin{array}{l}\text { Treatment with } \\
\text { antidepressants }\end{array}$ & - & N06A \\
\hline Treatment with anxiolytics & - & N05BA \\
\hline
\end{tabular}

${ }^{a}$ Danish National Patient Registry, based on the International Classification of Diseases 10

${ }^{b}$ Danish National Prescription Registry, based on the Anatomical Therapeutic Chemical classification system. 
Table 2 Characteristics of the study populations.

\begin{tabular}{|c|c|c|c|c|}
\hline & \multicolumn{2}{|c|}{ Singletons } & \multicolumn{2}{|c|}{ Twins $^{a}$} \\
\hline & Cases & Controls & Cases & Controls \\
\hline Number & 2631 & 10524 & 375 & 375 \\
\hline Mean age (years) & 67 & 67 & 64 & 64 \\
\hline $\begin{array}{l}\text { Mean age at } \\
\text { diagnosis (years) }\end{array}$ & 61 & & 56 & \\
\hline Females (\%) & 81 & 81 & 82 & 82 \\
\hline $\begin{array}{l}\text { Mean follow-up } \\
\text { duration (years) }\end{array}$ & 6 & 6 & 6 & 6 \\
\hline $\mathrm{CS}=0(\%)$ & 58 & 65 & 64 & 67 \\
\hline$C S=1(\%)$ & 23 & 20 & 18 & 20 \\
\hline$C S>1(\%)$ & 20 & 15 & 17 & 13 \\
\hline
\end{tabular}

CS, Charlson score.

${ }^{\mathrm{a}}$ Twins from pairs discordant for hyperthyroidism.

\section{Results}

\section{Baseline characteristics of the study populations}

The baseline characteristics of singletons and twins diagnosed with hyperthyroidism, as well as their controls, are given in Table 2 . The mean age of singletons and twins at the diagnosis of hyperthyroidism was 61 and 56 years respectively. Cases and controls were followed for an average of 6 years (range 0-13). In general, cases had a higher CS than their respective controls and twins had a lower CS than the singletons.

\section{Overall association between hyperthyroidism and psychiatric morbidity}

As summarized in Table 3, subjects with hyperthyroidism had, compared with the control population, a higher frequency of being hospitalized with psychiatric diagnoses (5 vs $3 \%$ respectively; $P=0.004$ ), which did not change significantly after subdividing into primary or secondary causes of hospitalization (data not shown). Hyperthyroid individuals also had a higher risk of being treated with antipsychotics, antidepressants, or anxiolytics.

\section{Psychiatric morbidity before the diagnosis of hyperthyroidism}

Singletons with hyperthyroidism had an increased risk of being hospitalized with psychiatric diagnoses (OR: 1.33; 95\% CI: 0.98-1.80) before the diagnosis of hyperthyroidism. This was also true when psychiatric disease was the primary cause of hospitalization (OR: 1.50; 95\% CI: 0.99-2.27). In addition, hyperthyroid individuals, when compared with their controls, were more often treated with antipsychotics
(OR: 1.17; 95\% CI: 1.00-1.38), antidepressants (OR: 1.13; 95\% CI: 1.01-1.27), or anxiolytics (OR: 1.28; 95\% CI: 1.16-1.42) before the diagnosis of hyperthyroidism (Table 3). After controlling for the educational level, these values did not change significantly. Stratification for the cause of hyperthyroidism revealed only Graves' disease (GD) (OR: 1.85; 95\% CI: 1.24-2.60) but not toxic nodular goiter (TNG) (OR: 1.33; 95\% CI: 0.83-2.15) to be associated with a significantly increased risk of hospitalization with a psychiatric diagnosis before the diagnosis of hyperthyroidism. Evaluation of the same disease categories, but censoring of diagnoses made 365 days before the diagnosis of hyperthyroidism, yielded essentially similar results, although the results for treatment with antidepressants were attenuated (OR: 1.00; 95\% CI: 0.88-1.13; Table 3).

\section{Psychiatric morbidity following the diagnosis of hyperthyroidism}

The hazard ratios (HRs) for psychiatric morbidity after the diagnosis of hyperthyroidism are given in Table 3. Singletons with hyperthyroidism had an increased risk of being hospitalized with psychiatric diagnoses (HR: 1.51; 95\% CI: 1.11-2.05) and treated with antipsychotics (HR: 1.46; 95\% CI: 1.20-1.79), antidepressants (HR: 1.54; 95\% CI: 1.36-1.74), or anxiolytics (HR: 1.47; 95\% CI: 1.27-1.69), following the diagnosis of hyperthyroidism. Controlling for the educational level did not change these findings. Also when psychiatric disease was the primary cause of hospitalization, hyperthyroid individuals had a higher risk of being hospitalized due to psychiatric diagnoses afterwards (HR: 1.52; 95\% CI: 0.99-2.35). Except for hospitalization with psychiatric diagnoses, which was attenuated (HR: 1.18; 95\% CI: 0.82-1.69), these results did not change significantly when censoring diagnoses made 365 days after the diagnosis of hyperthyroidism (Table 3). Also competing risk had only a minor impact, as hyperthyroidism remained significantly associated with treatment with antipsychotics, antidepressants, and anxiolytics in these analyses. However, stratification for the cause of hyperthyroidism revealed only GD (OR: 2.23; 95\% CI: 1.30-3.83) but not TNG (OR: 0.99; 95\% CI: $0.50-1.94)$ to be associated with a significantly increased risk of hospitalization with a psychiatric diagnosis following the diagnosis of hyperthyroidism.

\section{Psychiatric morbidity in twin pairs discordant for hyperthyroidism}

In a combined analysis based on being hospitalized with psychiatric diagnoses and/or treated with antipsychotics, 
Table 3 Extent and temporality of psychiatric morbidity in hyperthyroidism.

\begin{tabular}{l} 
Disease category \\
\hline $\begin{array}{c}\text { Hospitalization with } \\
\text { psychiatric diagnoses }\end{array}$
\end{tabular}

Treatment with antipsychotics

Treatment with antidepressants

Treatment with anxiolytics

\begin{tabular}{|c|c|c|}
\hline \multicolumn{3}{|c|}{ Prevalence in percentage } \\
\hline Cases (number) & Controls (number) & $P$ values \\
\hline $5 \%(119)$ & $3 \%(353)$ & 0.004 \\
\hline
\end{tabular}

$14 \%(357)$

$11 \%(1167)$

$<0.001$

$33 \%(855)$

$39 \%(1023)$

$32 \%(3342)$

\begin{tabular}{c}
\hline Odds ratios $^{\mathrm{a}}$ \\
\hline $1.33(0.98-1.80)$ \\
$1.25(0.88-1.76)^{\mathrm{c}}$ \\
$1.16(0.83-1.64)^{\mathrm{d}}$ \\
$1.17(1.00-1.38)$ \\
$1.10(0.91-1.33)^{\mathrm{c}}$ \\
$1.16(0.98-1.38)^{\mathrm{d}}$ \\
$1.13(1.01-1.27)$ \\
$1.10(0.97-1.25)^{\mathrm{c}}$ \\
$1.00(0.88-1.13)^{\mathrm{d}}$ \\
$1.28(1.16-1.42)$ \\
$1.25(1.12-1.40)^{\mathrm{c}}$ \\
$1.21(1.09-1.34)^{\mathrm{d}}$ \\
\end{tabular}

Hazard ratios ${ }^{\mathrm{b}}$

$1.51(1.11-2.05)$

$1.47(1.03-2.11)^{\mathrm{c}}$

$1.18(0.82-1.69)^{\mathrm{d}}$

$1.08(0.76-1.53)^{\mathrm{e}}$

$1.46(1.20-1.79)$

$1.27(1.00-1.62)^{\mathrm{c}}$

$1.35(1.08-1.69)^{\mathrm{d}}$

$1.29(1.04-1.59)^{\mathrm{e}}$

1.54 (1.36-1.74)

$1.51(1.32-1.73)^{\mathrm{C}}$

$1.28(1.11-1.48)^{\mathrm{d}}$

$1.23(1.08-1.41)^{\mathrm{e}}$

1.47 (1.27-1.69)

$1.38(1.18-1.62)^{\mathrm{c}}$

$1.31(1.11-1.54)^{\mathrm{d}}$

$1.23(1.05-1.44)^{\mathrm{e}}$

adds ratios before the diagnosis of hyperthyroidism, adjusted for the Charlson score.

${ }^{\mathrm{b}}$ Hazard ratios after the diagnosis of hyperthyroidism, adjusted for the Charlson score.

'Adjusted for the Charlson score and educational level categorized into primary, secondary, and tertiary.

d 365 -day censoring window before and after the diagnosis of hyperthyroidism.

émpeting risk.

antidepressants, or anxiolytics, we found significantly increased psychiatric morbidity in the hyperthyroid twins than in the euthyroid co-twins (OR: 1.70; 95\% CI: 1.20-2.41). After stratification for zygosity, there was no significant difference in the risk estimates between dizygotic (DZ) twins (OR: 1.70; 95\% CI: 1.10-2.61) and monozygotic (MZ) twins (OR: 1.71; 95\% CI: 0.94-3.10). Due to the lack of power, further subdivision, according to whether treated in a hospital setting and type of psychiatric drug, was not meaningful.

\section{Discussion}

By utilizing population-based Danish health registers, we were able to investigate a large sample size with validated definitions of thyroid disease $(20,27)$ and well-accepted definitions of psychiatric outcome $(20,27,33,34,35)$. We found an overall positive association between hyperthyroidism, on the one hand, and hospitalization with psychiatric diagnoses as well as treatment with antipsychotics, antidepressants, or anxiolytics, on the other hand. Moreover, our design allowed for the evaluation and demonstration of an increased risk of being hospitalized with psychiatric diagnoses and an increased risk of being treated with antipsychotics, antidepressants, and anxiolytics, both before and after the diagnosis of hyperthyroidism. These findings indicate a direct association between hyperthyroidism and subsequent psychiatric morbidity and vice versa. Consistent with our findings, two Danish large-scale studies, based on hospital treatment, have reported an increased risk of affective disorders following the diagnosis of hyperthyroidism (20) and vice versa (27). The pattern of an increased risk of psychiatric morbidity both before and after the diagnosis of hyperthyroidism could be explained by the presence of shared genetic factors associated with psychiatric morbidity and hyperthyroidism. Both hyperthyroidism $(28,29)$ and various psychiatric morbidities $(30,31,32)$ demonstrate familial aggregation. If these conditions cross-trait, the finding of an increased risk of psychiatric morbidity associated with hyperthyroidism could be due to genetic confounding. However, based on our findings within twin pairs discordant for hyperthyroidism, genetic confounding is unlikely to explain the increase in psychiatric morbidity, as risk estimates are unaffected by stratification for zygosity.

It is important to note that this association between hyperthyroidism and psychiatric morbidity could also be explained by the presence of shared risk factors, such as negative live events, suggested to be associated with both hyperthyroidism (37) and psychiatric diseases (38). Unfortunately, we were unable to explore this issue further.

The observation that the detection of one disease leads to a higher rate of detection of other and not necessarily related diseases, denoted Berkson's bias, is well accepted 
(39) and might constitute another reason for the increased risk of psychiatric morbidity both before and after the diagnosis of hyperthyroidism. To minimize this detection bias, we censored diagnoses made 365 days before and after the diagnosis of hyperthyroidism. Applying this censoring, the risk of being hospitalized with psychiatric diagnoses was found to be attenuated, indicating that detection bias might explain the observed association. By contrast, when considering the risk of being treated with antipsychotics, antidepressants, and anxiolytics, our data suggest Berkson's bias to be less likely.

Hyperthyroidism is most often caused by autoimmunity, as in GD, or by one or more autonomously functioning thyroid nodules, as in TNG (40). While GD and TNG differ with respect to age at onset, symptoms, and duration (41), the majority of studies evaluating the association between hyperthyroidism and psychiatric diseases do not differentiate between the causes of thyroid dysfunction. It has been suggested that the increased risk of psychiatric morbidity observed in subjects with thyroid autoimmunity could be due to autoimmunity per se rather than due to the thyroid state. In line with this, a variety of autoimmune diseases (19), including thyroid autoimmunity $(19,20)$, have been reported to be associated with an increased risk of psychiatric morbidity. In the present study, when stratifying for the cause of disease (GD or TNG), the higher risk of being hospitalized with psychiatric diagnoses following the diagnosis of hyperthyroidism was completely attenuated in TNG individuals, but not in GD individuals. Accordingly, these data are supportive of psychiatric diseases being attributed to autoimmunity rather than to the hyperthyroid state. By contrast, when considering the risk of being treated with antipsychotics, antidepressants, and anxiolytics, our data suggest no difference between GD and TNG. Still, the exact pathophysiological mechanisms related to the possible difference in psychiatric morbidity associated with GD and TNG require further investigations.

The strengths of the present study include a large sample size, ascertainment of participants from nationwide population-based registers, a relatively long observation period, and the possibility of stratifying for the time period before and after the diagnosis of hyperthyroidism. Importantly, we minimized the risk of selection bias, as hyperthyroid cases were identified from both the DNPR and DNPrR, and consequently both patients from a hospital setting - whether outpatients or inpatients - and patients from primary care were accounted for. However, the lack of information regarding the type of hyperthyroid therapy and effect of treatment and the lack of biochemical data are weaknesses of the present study hindering any conclusion regarding the association between hyperthyroidism and psychiatric morbidity.

To our knowledge, this is the first study to identify psychiatric diseases due to hospitalizations with ICD diagnoses (DNPR) and registration of pharmacological treatment (DNPrR). Although both methods are well accepted $(20,27,33,34,35)$, the use of pharmacological treatment as a proxy for psychiatric morbidity raises some questions. Despite an expected high sensitivity of the DNPrR for psychiatric diseases, the specificity is lower as, for example, TCAs may be used in the treatment of pain. In addition, as hyperthyroidism may mimic panic attacks, anxiolytics may be prescribed for these conditions. As a consequence, we are likely to have overestimated the frequency of psychiatric morbidity in the present study in both cases and controls. By contrast, the DNPR has been validated for various psychiatric diagnoses, e.g. schizophrenia and depression (42). Still, it is comforting that, whether psychiatric diseases were detected from the DNPR - and whether focusing on primary or secondary diagnoses - or from the DNPrR, our results are consistent and the risk of bias is therefore limited.

We conclude that hyperthyroidism is associated with a higher risk of being hospitalized with or being pharmacologically treated for psychiatric diseases, both before and after the diagnosis of hyperthyroidism. While detection bias cannot be completely ruled out, we could not detect any genetic confounding.

\section{Declaration of interest}

The authors declare that there is no conflict of interest that could be perceived as prejudicing the impartiality of the research reported.

\section{Funding}

F Brandt and $M$ Thvilum are enrolled as PhD students financed by the School of Endocrinology, University of Southern Denmark in Odense. $\mathrm{F}$ Brandt and $\mathrm{M}$ Thvilum also received funding from the Danish Thyroid Patient Organization. L Hegedüs is the recipient of an unrestricted research grant from the Novo Nordisk Foundation.

\section{References}

1 Bernal J. Thyroid hormone receptors in brain development and function. Nature Clinical Practice. Endocrinology \& Metabolism 20073 249-259. (doi:10.1038/ncpendmet0424)

2 Guandano-Ferraz A, Obregon MJ, St-Germanin D \& Bernal J. The type 2 iodothyronine deidonase is expressed primarily in glial cells in the 
neonatal rat brain. PNAS 199794 10391-10396. (doi:10.1073/ pnas.94.19.10391)

3 Hernandez A, Martinez ME, Fiering S, Galton VA \& St Germanin D. Type 3 deiodinase is critical for the maturation and function of the thyroid axis. Journal of Clinical Investigation 2006116 476-484. (doi:10.1172/JCI26240)

4 Haddow JE, Palomaki GE, Allan WC, Williams JR, Knight GJ, Gagnon J, O'Heir CE, Mitchell ML, Hermos RJ, Waisbren SE et al. Maternal thyroid deficiency during pregnancy and subsequent neuropsychological development in the child. New England Journal of Medicine 1999341 549-555. (doi:10.1056/NEJM199908193410801)

5 Watt T, Groenvold M, Rasmussen AK, Bonnema SJ, Hegedüs L, Bjorner JB \& Feldt-Rasmussen U. Quality of life in patients with benign thyroid disorders. A review. European Journal of Endocrinology $2006 \mathbf{1 5 4}$ 501-510. (doi:10.1530/eje.1.02124)

6 Bauer M, Goetz T, Glenn T \& Whybrow PC. The thyroid-brain interaction in thyroid disorders and mood disorders. Journal of Neuroendocrinology 200820 1101-1114. (doi:10.1111/j.1365-2826. 2008.01774.x)

7 Krishnan V \& Nestler EJ. The molecular neurobiology of depression. Nature 2008455 894-902. (doi:10.1038/nature07455)

8 Argyropoulus SV, Bell CJ \& Nutt DJ. Brain function in social anxiety disorder. Psychiatric Clinics of North America 200124 707-722. (doi:10.1016/S0193-953X(05)70259-8)

9 Brandt F, Thvilum M, Almind D, Christensen K, Green A, Hegedüs L \& Brix TH. Morbidity before and after the diagnosis of hyperthyroidism. A nationwide register-based study. PLoS ONE 20138 e66711. (doi:10.1371/journal.pone.0066711)

10 Thvilum M, Brandt F, Almind D, Christensen K, Brix TH \& Hegedüs L. Type and extent of morbidity before and after the diagnosis of hypothyroidism. A nationwide register study. PLOS ONE 20138 e75789. (doi:10.1371/journal.pone.0075789)

11 Thvilum M, Brandt F, Brix TH \& Hegedüs L. A review of the evidence for and against increased mortality in hypothyroidism. Nature Reviews. Endocrinology 20126 417-424. (doi:10.1038/nrendo.2012.29)

12 Thvilum M, Brandt F, Almind D, Christensen K, Hegedüs L \& Brix TH. Excess mortality in patients diagnosed with hypothyroidism. A nationwide cohort study of singletons and twins. Journal of Clinical Endocrinology and Metabolism 201398 1069-1075. (doi:10.1210/ jc. 2012-3375)

13 Brandt F, Green A, Hegedüs L \& Brix TH. A critical review and meta-analysis of the association between overt hyperthyroidism and mortality. European Journal of Endocrinology 2011165 491-497. (doi:10.1530/EJE-11-0299)

14 Brandt F, Almind D, Christensen K, Green A, Brix TH \& Hegedüs L. Excess mortality in hyperthyroidism: the influence of preexisting comorbidity and genetic confounding: a Danish nationwide registerbased cohort study of twins and singletons. Journal of Clinical Endocrinology and Metabolism 201297 4123-4129. (doi:10.1210/ jc. 2012-2268)

15 Brandt F, Thvilum M, Almind D, Christensen K, Green A, Hegedüs L \& Brix TH. Graves' disease and toxic nodular goiter are both associated with increased mortality but differ with respect to the cause of death: a Danish population-based register study. Thyroid 201323 408-413. (doi:10.1089/thy.2012.0500)

16 Williams MD, Harris R, Dayan CM, Evans J, Gallacher J \& Ben-Shlomo Y. Thyroid function and the natural history of depression: findings from the Caerphilly Prospective Study (CaPS) and a metaanalysis. Clinical Endocrinology 200970 484-492. (doi:10.1111/ j.1365-2265.2008.03352.x)

17 Pop VJ, Maartens LH, Leusink G, van Son MJ, Knottnerus AA, Ward AM, Metcalfe R \& Weetman AP. Are autoimmune thyroid dysfunction and depression related. Journal of Clinical Endocrinology and Metabolism 1998 83 3194-3197. (doi:10.1210/jc.83.9.3194)

18 Carta MG, Loviselli A, Hardoy MC, Massa S, Cadedda M, Sardu C, Carpiniello B, Dell'Osso L \& Mariotti S. The link between thyroid autoimmunity (antithyroid peroxidase autoantibodies) with anxiety and mood disorders in the community: a field of interest for public health in future. BMC Psychiatry 2004425 . (doi:10.1186/ 1471-244X-4-25)

19 Eaton WW, Pedersen MG, Nielsen PR \& Mortensen PB. Autoimmune diseases, bipolar disorder, and non-affective psychosis. Bipolar Disorders 201012 638-646. (doi:10.1111/j.1399-5618.2010.00853.x)

20 Thomsen AF, Kvist TK, Andersen PK \& Kessing LV. Increased risk of affective disorder following hospitalization with hyperthyroidism a register-based study. Thyroid 2005152 535-543.

21 Whybrow PC, Prange AJ \& Treadway CR. Mental changes accompanying thyroid gland dysfunction. A reappraisal using objective psychological measurement. Archives of General Psychiatry 196920 48-63. (doi:10.1001/archpsyc.1969.01740130050004)

22 Bunevicius R \& Prange AJ. Thyroid disease and mental disorders: cause and effect or only comorbidity? Current Opinion in Psychiatry 201023 363-368. (doi:10.1097/YCO.0b013e3283387b50)

23 Samuels MH. Cognitive function in untreated hypothyroidism and hyperthyroidism. Current Opinion in Endocrinology, Diabetes, and Obesity 200815 429-433. (doi:10.1097/MED.0b013e32830eb84c)

24 Engum A, Bjøro T, Mykletum A \& Dahl AA. An association between depression, anxiety and thyroid function - a clinical fact or an artefact. Acta Psychiatrica Scandinavica 2002106 27-34. (doi:10.1034/ j.1600-0447.2002.01250.x)

25 Engum A, Bjøro T, Mykletum A \& Dahl AA. Thyroid autoimmunity, depression and anxiety; are there any connections? An epidemiological study of a large population Journal of Psychosomatic Research 200559 263-268. (doi:10.1016/j.jpsychores.2005.04.002)

26 Sauvage MF, Marquet P, Rousseau A, Raby C, Buxeraud J \& Lachatre G. Relationship between psychotropic drugs and thyroid function: a review. Toxicology and Applied Pharmacology 2008149 127-135. (doi:10.1006/taap.1998.8367)

27 Thomsen AF \& Kessing LV. Increased risk of hyperthyroidism among patients hospitalized with bipolar disorder. Bipolar Disorders 20057 351-357. (doi:10.1111/j.1399-5618.2005.00205.x)

28 Brix TH, Kyvik KO, Christensen K \& Hegedüs L. Evidence for a major role of heredity in Graves' disease: a population-based study of two Danish twin cohorts. Journal of Clinical Endocrinology and Metabolism 200186 930-934. (doi:10.1210/jc.86.2.930)

29 Brix TH, Kyvik KO \& Hegedüs L. Major role of genes in the etiology of simple goiter in females: a Population-Based Twin Study. Journal of Clinical Endocrinology and Metabolism 199984 3071-3075. (doi:10.1210/ jc.84.9.3071)

30 Domschke K \& Dechert J. Genetics of anxiety disorders - status qou and qou vadis. Current Pharmaceutical Design 201218 5691-5698. (doi:10.2174/138161212803530781)

31 McGuffin P, Perroud N, Uher R, Butler A, Aitchinson KJ, Craig I, Lewis C \& Farmer A. The genetics of affective disorders and suicide. European Psychiatry 201025 275-277. (doi:10.1016/j.eurpsy.2009.12.012)

32 Sullivan PF, Kendler KS \& Neale MC. Schizophrenia as a complex trait: evidence from a meta-analysis of twin studies. Archives of General Psychiatry 200360 1187-1192. (doi:10.1001/archpsyc.60.12.1187)

33 Ekedahl A, Lidbeck J, Lithman T, Noreen D \& Melander A. Benzodiazepine prescribing patterns in a high-prescribing Scandinavian community. Clinical Pharmacology 199344 141-146. (doi:10.1007/BF00315471)

34 Nakagawa S, Pedersen L, Olsen ML, Mortensen PB, Sørensen HT \& Johnsen SP. Antipsychotics and risk of first-time hospitalization for myocardial infarction: a population-population case-control study. Journal of Internal Medicine 2006260 451-458. (doi:10.1111/ j.1365-2796.2006.01708.x)

35 Suppli NP, Deltour I, Damkjær LH, Christensen J, Jensen AB, Kroman NT, Johansen C \& Dalton SO. Factors associated with the prescription of antidepressive medication to breast cancer patients. Acta Oncologica 201150 243-251. (doi:10.3109/0284186X. 2010.531049) 
36 Madsen M, Andersen PK, Gerster M, Nybo Andersen AM, Christensen K $\&$ Osler M. Does the association of education with breast cancer replicate within twin pairs? A register-based study on Danish female twins. British Journal of Cancer $2011 \mathbf{1 0 4}$ 520-523. (doi:10.1038/ sj.bjc.6606090)

37 Bagnasco M, Bossert I \& Pesce G. Stress and autoimmune thyroid disease. Neuroimmunomodulation 200613 309-317. (doi:10.1159/ 000104859)

38 Kendler KS, Karkowski LM \& Prescott CA. Causal relationship between stressful life events and the onset of major depression. American Journal of Psychiatry 1999156 837-841.
39 Berkson J. Limitations of the application of fourfold table analysis to hospital data. Biometrics Bulletin 19462 126-135. (doi:10.2307/ 3002000)

40 Franklyn JA \& Boelaert K. Thyrotoxicosis. Lancet 2012379 1155-1166. (doi:10.1016/S0140-6736(11)60782-4)

41 Biondi B \& Kahaley GJ. Cardiovascular involvement in patients with different causes of hyperthyroidism. Nature Reviews. Endocrinology 20106 431-443. (doi:10.1038/nrendo.2010.105)

42 Mors O, Perto GP \& Mortensen PB. The Danish psychiatric central research registers. Scandinavian Journal of Public Health 201139 54-57. (doi:10.1177/1403494810395825)

Received 27 August 2013

Revised version received 7 November 2013

Accepted 26 November 2013 